\title{
Filter methods to preserve local contrast and to avoid artifacts in gamut mapping
}

\author{
Marcel Meili, Dennis Küpper, Zofia Barańczuk, Ursina Caluori, Klaus Simon \\ Swiss Federal Laboratories for Materials Testing and Research (EMPA), Dübendorf, Switzerland
}

\begin{abstract}
Contrary to high dynamic range imaging, the preservation of details and the avoidance of artifacts is not explicitly considered in popular color management systems. An effective way to overcome these difficulties is image filtering. In this paper we investigate several image filter concepts for detail preservation as part of a practical gamut mapping strategy. In particular we define four concepts including various image filters and check their performance with a psycho-visual test. Additionally, we compare our performance evaluation to two image quality measures with emphasis on local contrast. Surprisingly, the most simple filter concept performs highly efficient and achieves an image quality which is comparable to the more established but slower methods.
\end{abstract}

Keywords: Color Appearance, Gamut Mapping, Image Quality Measures, Psycho-visual Tests

\section{INTRODUCTION}

In the early nineties the WYSIWYG-principle of desktop publishing determined color correctness as the primary target of image reproduction. Accordingly, the 1993 founded and still dominating ICC-Color Management System (ICC-CMS), see www . color . org, is primarily designed as color transformation. The necessary adaption to the limited possibilities of printing color spaces, so-called gamut mapping, was defined as a static point-to-point function.

However, in ICC-CMS, the specification of gamut mapping remained open and was left to the software producers. Hence the search for an universal gamut mapping algorithm became an open problem in color community, see CIE2004. ${ }^{1}$ Recent results show that image dependent methods, e.g. the treatment of image colors as source gamut, lead to visually significantly improved algorithms in terms of image quality. This directed the attention to the question whether other imaging concepts could be useful for gamut mapping, in particular, filter based methods. The focus of this gamut mapping concept is rather on image quality then color correctness and should be understand as a paradigm shift in the image reproduction.

A related area to gamut mapping is tone mapping of HDR images, see Reinhard. ${ }^{2}$ In this context multi-scale image representations are well-established. Thereby the image is decomposed by a filter bank in different frequency banks (see e.g. Yuanzhen ${ }^{3}$ ) similar to a wavelet decomposition (see e.g. Mallat ${ }^{4}$ ). Corresponding papers investigating this concept in gamut mapping are Morovic ${ }^{5}$ and Farup. ${ }^{6}$ Accordingly, a spatial invariant gamut mapping $G M$ is used to iteratively construct an image sequence $f_{0}, f_{1}, \ldots, f_{p}$ beginning with the original image $f_{0}$. The sequence is defined as:

$$
f_{i+1}=G M\left(\text { low-pass }\left(f_{i}\right)\right) \oplus \underbrace{f_{i}-\text { low-pass }\left(f_{i}\right)}_{\text {high-pass filter }\left(f_{i}\right)}
$$

The advantage of this approach is a high-contrast image with a good detail preservation at every scale. Drawbacks are long execution times and a tendency to show artifacts.

Instead of using filters for the core of the gamut mapping algorithm, they can be used in addition and for improving conventional gamut mapping. Bala ${ }^{7,8}$ uses Gauss-filters which are applied to the difference $O-G M(O)$, where $\mathrm{O}$ is the original image and $G M(O)$ the gamut mapped image. The adding of the filtered image reconstructs many of the details lost during gamut mapping. Zolliker ${ }^{9}$ replaces the Gaussian filter by an edge-preserving smoothing filter to avoid unwanted halos at sharp edges and addressing another range (frequency) of details. Further it was shown that all considered gamut mapping algorithms, especially the CIE-reference algorithms HPminDist and SGCK, benefit more or less in a same amount of this improvement.

Color Imaging XV: Displaying, Processing, Hardcopy, and Applications, edited by Reiner Eschbach, Gabriel G. Marcu, Shoji Tominaga, Alessandro Rizzi, Proc. of SPIE-IS\&T Electronic Imaging, SPIE Vol. 7528, 75280I - (c) 2010 SPIE-IS\&T CCC code: $0277-786 X / 10 / \$ 18 \cdot$ doi: $10.1117 / 12.838974$ 
A natural alternative strategy to the above described detail reconstruction is, of course, avoiding their loss and this is the topic of this paper. In general, we start by extracting details from the difference between the original and the filtered image. Then we apply the gamut mapping to the smoothed image instead of the original. Finally, details are added back to the mapped image. Due to this approach, details are not lost to a large extent because of they are not involved in the gamut mapping. Artifacts caused by geometrical discontinuities of the target gamut are reduced, since generally filtering brings neighboring pixel together and closer colors are mapped closer together.

In section 3 we introduce our algorithms and the employed filter, section 4 explains the psycho-visual test and section 5 is covered with the discussion of our results.

\section{ALGORITHMS}

This section includes the various combinations of filters and algorithms involved in our test. As realization of a spatial invariant GM-algorithm we select a simple basic concept to minimize the mutual influence of filter and gamut mapping. It can be describe as follows where $f_{O}$ stands for the original image, $f_{M}$ for the mapped image, $L P$ for a low-pass filter and $\oplus$ for the addition of the difference image:

$$
f_{M}=G M\left(L P\left(f_{O}\right)\right) \oplus\left(f_{O}-L P\left(f_{O}\right)\right)
$$

In other words, we consider only the first step of (1). Our objectives is an identification of filter-GM combination, featuring similar properties as Zolliker, but going along with a smaller filter size and a high visual performance. In an industrial workflow often a 1-pass-strategy is preferred, which in our case is the application of the filter taking place before the actual gamut mapping. The preliminar filtering of the original image has the additional advantage that, in general, halo artifacts do not have to be considered when using small filter sizes. The targeted 1-pass-strategy implies, that the filter is applied only once. With this, the carefully designed procedures to control the gain and to avoid artifacts in multi-scale approaches can be omitted to a large extent. However, the two basic questions of the multi-scale concept remain:

- Which filter shall be used?

- How shall the $\oplus$-operation be implemented i.e. in which way the addition of the difference image shall be realized?

According to the first question we consider:

1. a small Mean filter

2. a small Gauss filter

3. a small edge-preserving smoothing filter

4. a large edge-preserving smoothing filter

Since we try to design a concept suited for industrial purpose our solution shall perform well in regard of computation time but still having a good quality. The first two filter chosen are a simple mean filter taking the average of all values in the window and a corresponding Gauss filter. These filters are fast and easy to implement, but when applied with large filter sizes, halo artifacts may appear. Therefore we restricted these filters to a small size. The third type of filter is an edge-preserving smoothing filter similar to the Gauss filter, but with an additional weighting of the involved color diffrences. The color distance from each pixel in the window to the current value is calculated in LAB. Finally we consider an edge-preserving smoothing filter with large filter size as visual reference.

We consider 4 variants of our basic algorithm which consists of five steps:

1. Filter a copy of the original image

2. Create a difference image by subtracting the smoothed image from the original 
3. Map the filtered image to the destination gamut

4. Add the difference image to the gamut mapped image

5. Apply clipping to destination gamut

In the first step, the image is filtered with a smoothing filter. Then a difference image is created by subtracting the filtered image from the original. This image contains the details (high frequencies) of the image. The filtered image (low frequencies) is mapped to the destination gamut (ISO newspaper). Since we use a device-to-device method, we compress a color according to the input device gamut which is sRGB.

The compression is carried out along a ray $R$ spanned by a fixed focus point $F$ in the middle of the output gray axis and the given color $C$. Let $I$ and $O$ be the intersection points of $R$ and the surface of the input and output gamut, respectively. Then $C$ is mapped to the point $C^{\prime}$ on $R$ at distance $D$ from $F$ where

$$
D=\left(1-\left(1-\frac{\overline{F C}}{\overline{F I}}\right)^{\alpha}\right) \cdot \overline{F O} .
$$

In the algorithms $1-3$ we use $\alpha=2.5$ while algorithm 4 is applied with $\alpha=1.0$ which correspondes to a linear mapping.

Accordingly, a color lying on the boundary of the sRGB gamut is always mapped to the boundary of the output gamut. and a color lying exactly on the focal point remains the same.

Next we add back the difference image in detail described below. Since the result may be out of gamut, we clip all out of gamut colors to the boundary with a minimum distance clipping algorithm preserving the hue of the colors.

\subsection{Algorithm 1}

Figure 1 illustrates how a color $c$ is mapped to color $c^{\prime}$ given the filtered value $m$. The compression of $m$ is done nonlinear as mentioned above resulting in the mapped filtered value $m^{\prime}$. The filtered value $m$ is compressed towards the focal point and the difference $c-m$ is added to $m^{\prime}$. For visual reasons, the difference $c-m$ is scaled by factor of 1.1 .
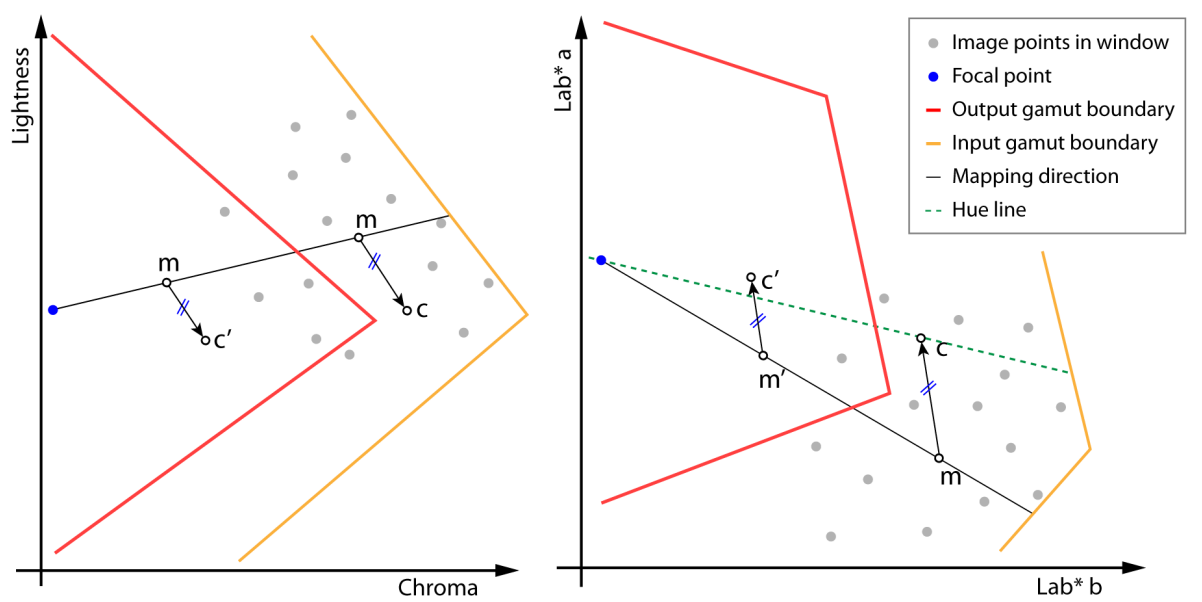

Figure 1. Left: Projection into the hue plane of color $c$. Right: Same scenario projected to onto a plane with constant lightness. The result $c^{\prime}$ may not have the same hue as the original value $c$

\subsection{Algorithm 2}

In the second algorithm we added an offset to the destination gamut boundary. The outmost color is not mapped to the boundary of the gamut, but closer to the center with an offset to the boundary. Therefore we shrink the destination gamut to $80 \%$ and performed the mapping. When the details are added back in step 4 , the chance is much higher that they are still in gamut. Hence less details are lost in the last step where out of gamut colors are clipped. Apart from that everything is the same as in algorithm 1. 


\subsection{Algorithm 3}

The third algorithm, illustrated in figure 2, adds details such that the original hue is preserved. First, the filtered value $m$ is mapped into the destination gamut using the reference algorithm, resulting in $m^{\prime}$. Both $m$ and $m^{\prime}$ are then rotated around the gray axis into the hue plane of the original color $c$, giving $m_{\text {hue }}$ and $m_{\text {hue }}^{\prime}$, respectively. The difference is now viewed as the relation between $\left|c-m_{\text {hue }}\right|$ and $\left|o-m_{\text {hue }}\right|$, where $o$ is the point on the gamut surface where one arrives when shooting a ray from $m_{\text {hue }}$ through $c$. This relation is amplified by transforming it non-linearly and adding it to $m_{\text {hue }}^{\prime}$ in an analogous way. When adding the transformed details in the destination gamut, we restrict their magnitude such that the differences do not exceed the differences in the original image. Additionally we used an offset as in algorithm 2 with a shrinkage to $90 \%$.
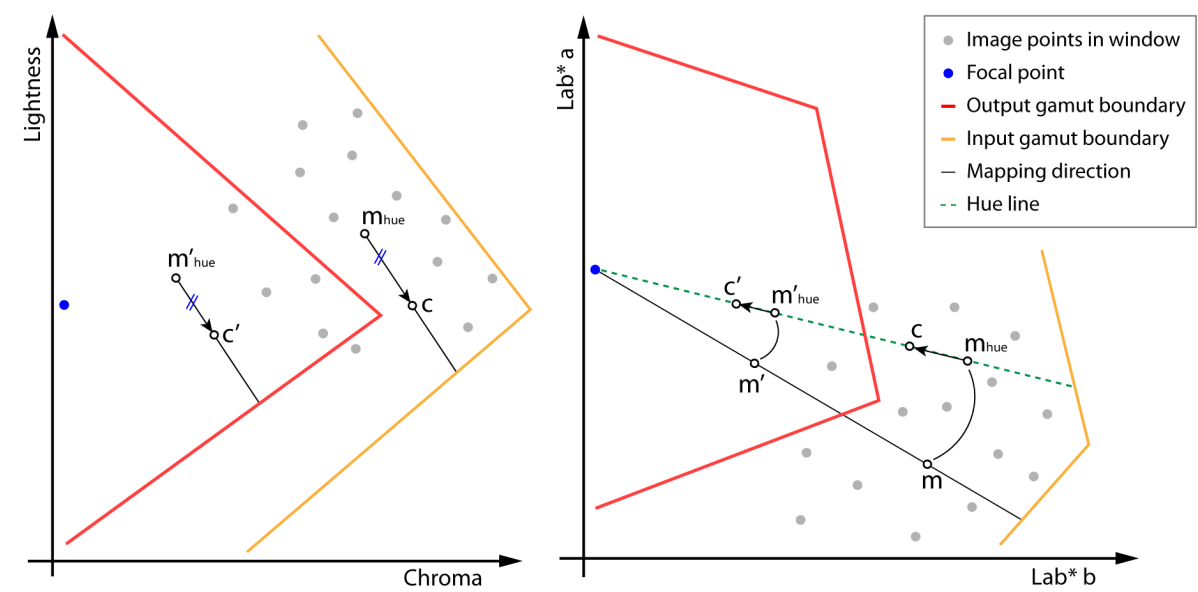

Figure 2. Left: Projection into the hue plane of color $c$. Right: Same scenario projected onto a plane with constant lightness. The result $c^{\prime}$ has the same hue as the original value $c$.

\subsection{Algorithm 4}

Algorithm 4 is similar to algorithm 3 with only two differences. There is no offset to the gamut boundary and we perform linear mapping instead of nonlinear mapping of $m$. In the algorithm 4 we try to translate the classic sigmoidal image reproduction technique to a filter based concept which is, compared to the other variants, a linear mapping for the GM. Apart from that it is the same as algorithm 3.

\section{PSYCHO-VISUAL TESTS}

For the evaluation of the visual performance of the algorithms introduced in section 3, we conducted several psychovisual tests.

\subsection{Setup}

We had four contrast enhancement algorithms with four different filter variants each, resulting in a total of 16 different methods to compare. Doing a naive pair comparison would result in a combinatorial nightmare of 120 comparisons per image. This is, even on our relatively small image set of 30 images, rather impractical. That is why we chose to break the test down into two tiers, see figure 3. In the first tier, we performed a separate pair comparison test for each algorithm, varying only the filter method within each test. In the second tier, the winners competed against each other, against Zolliker's contrast enhancement applied to our base algorithm, and against our base algorithm itself without any contrast enhancement.

The tests were performed by 11 observers on an image set of 30 images, in a standardized viewing environment, following the guidelines in the CIE technical report TC-08. ${ }^{10}$ The evaluation was done using Thurstone's method for evaluating pair comparison tests. In figures 4 and 5 you can see the image sets used for tier 1 and tier 2 tests respectively. Figures 6 and 7 show all the variants for one image of tier 1 and tier 2 respectively. 


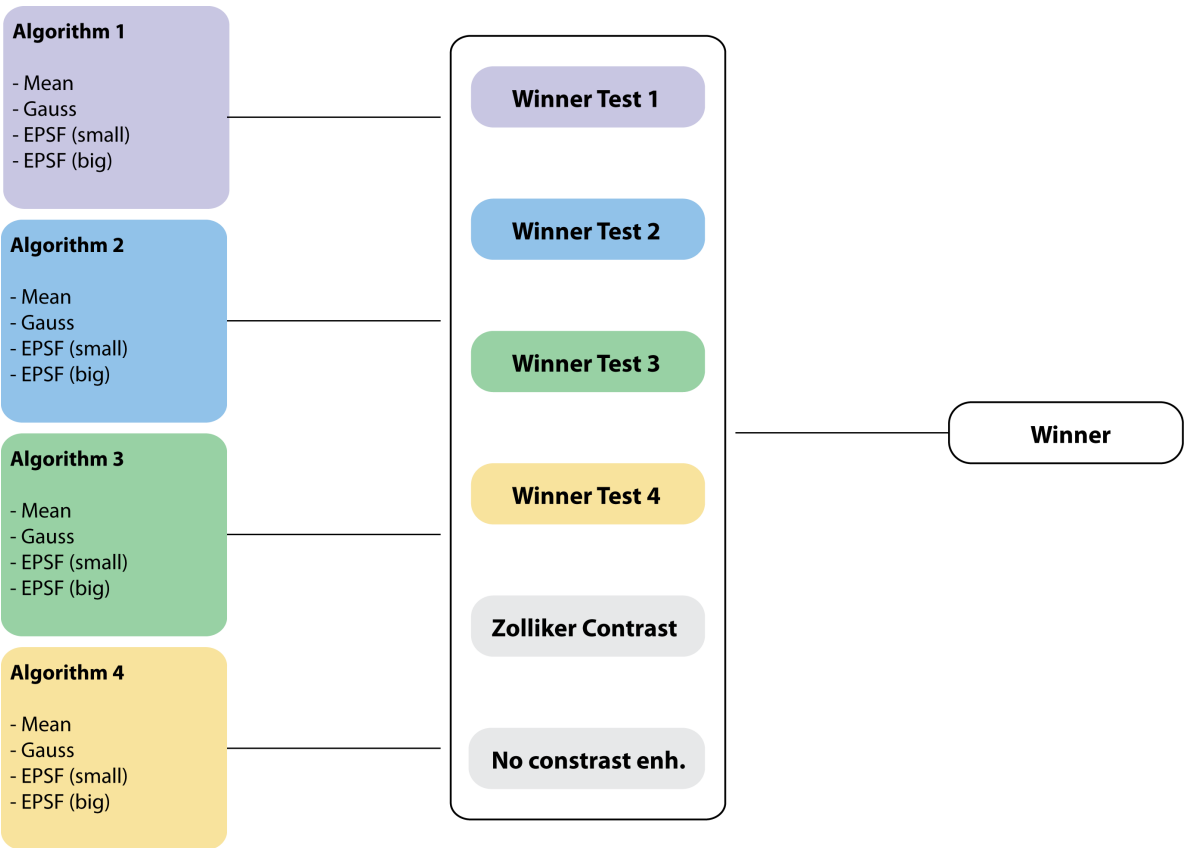

Figure 3. Test setup: The left column represents the first tier containing one test per algorithm. The best filter-algorithm combinations compete against each other and two reference algorithms in the second tier, yielding one winner in the end.

\subsection{Results}

Our goals for these tests were

- to verify that we had developed contrast enhancement algorithms that are better than our base algorithm.

- to verify that we could achieve comparable visual performance to Zolliker's algorithm applied to our base algorithm, but with significantly lower computational effort.

The base algorithm is a simplified version of the gamut mapping algorithm introduced in Caluori. ${ }^{11}$ Zolliker's method is an algorithm that is applied to already gamut-mapped images in order to enhance contrast. It yields highquality, high-detail images but it is also very slow. We developed four algorithms based on our base algorithm that do the contrast enhancement before the gamut mapping, thus maximizing the efficiency while still delivering good quality and details.

As you can see in figures 8 and 9, there was a clear winner in each of the first-tier tests. Surprisingly the simpler filters performed better than the more complex edge-preserving smoothing filters. Though unexpected, this is a nice revelation because it again reduces computational cost. The winners of the first tier were the Gauss filter for algorithms 1,2 and 4, and the mean filter for algorithm 3.

The green bars in figure 10 are the results of the final test. As hoped, they confirm that we have achieved both our goals: All of the new methods beat the base algorithm and method 1 with Gauss filter even beats Zolliker, even though just by a small margin.

\section{IMAGE QUALITY MEASURES}

The main topic of this paper is analyzing various filters preserving or enhancing details in the gamut mapping process. An interesting question is now how in this case the computed difference in local contrast $(\Delta L C)$ between original and mapped images correlates with the observer preferences evaluated in the psycho-visual test. The correlation between observer preferences in psycho-visual tests concerning gamut mapping algorithms and image quality measures 


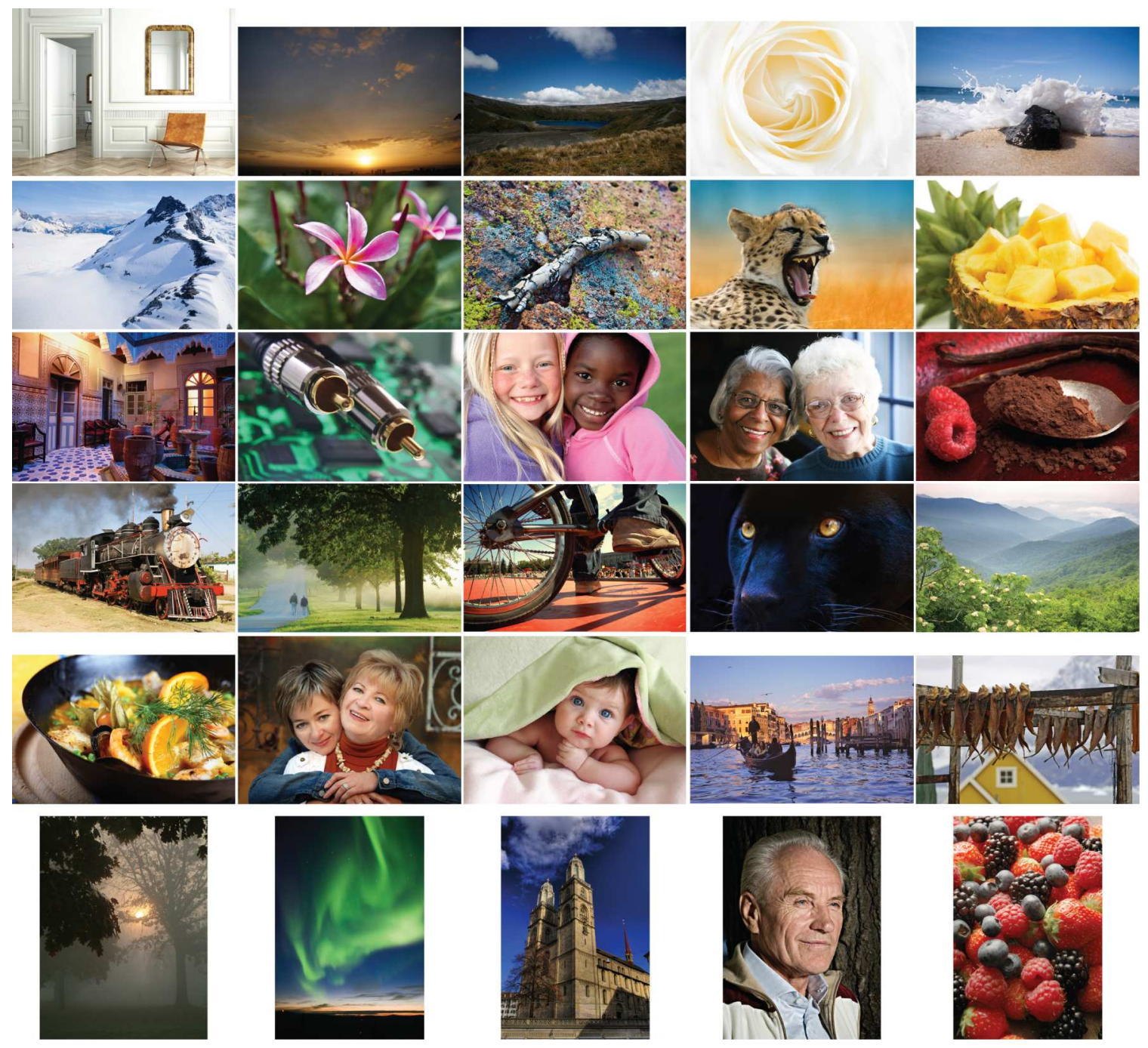

Figure 4 . Test set for the tier one tests. 

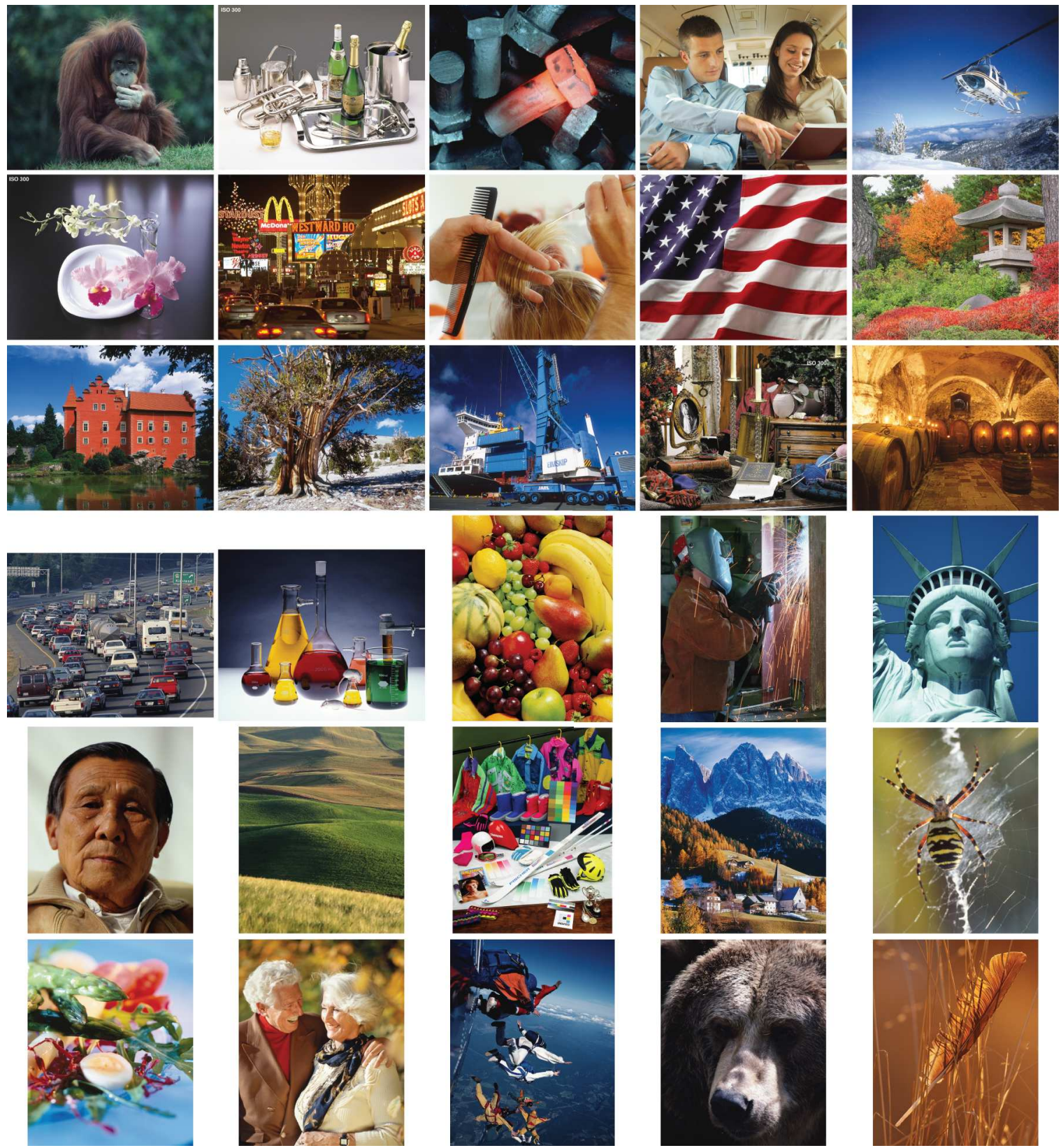

Figure 5. Test set for the tier two test.

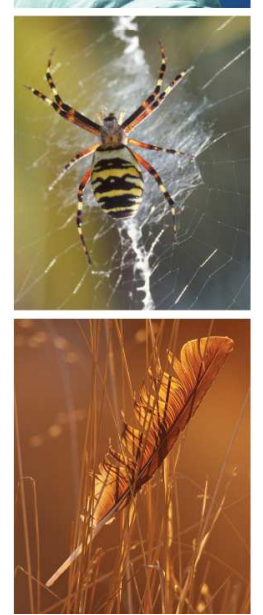


Algo 1
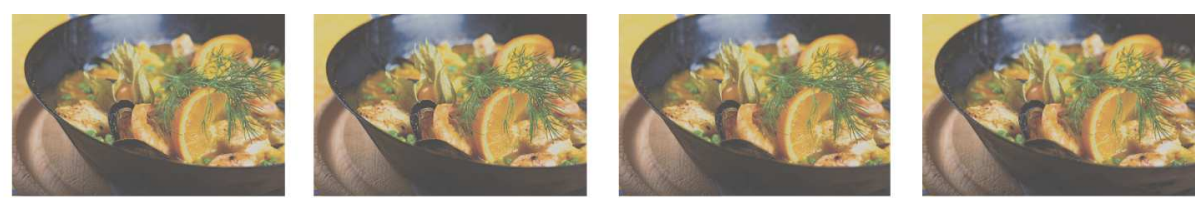

Algo 2
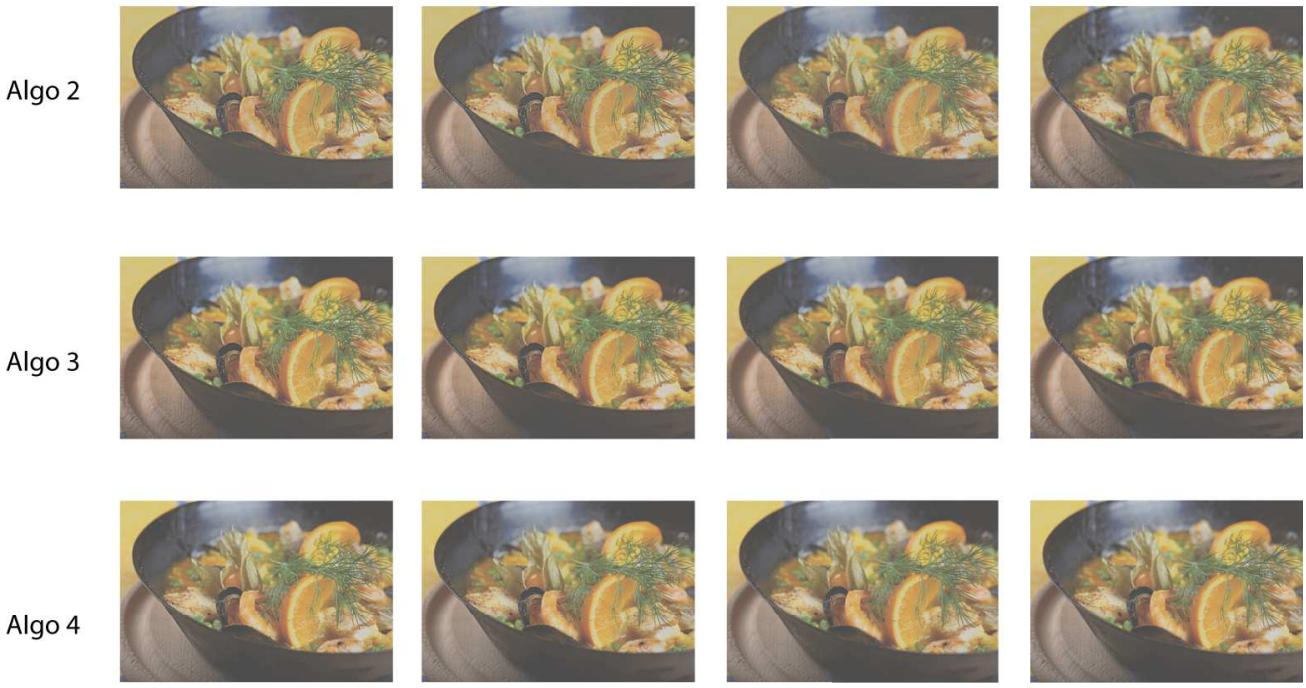

Mean

Gauss

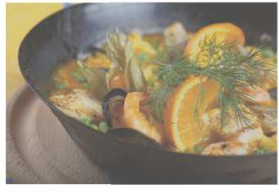

EPSF small

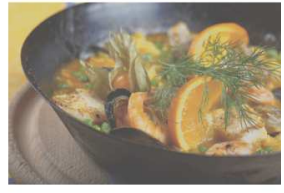

EPSF big

Figure 6. One image from our test set transformed with each of our 16 methods.

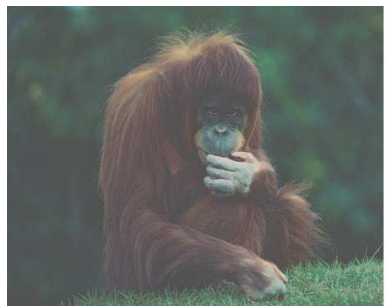

Winner 1

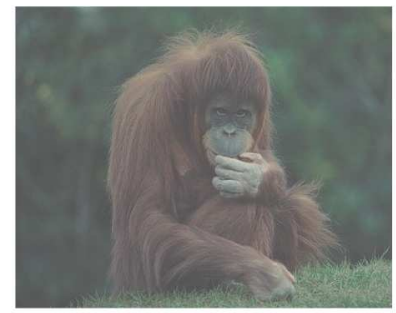

Winner 4

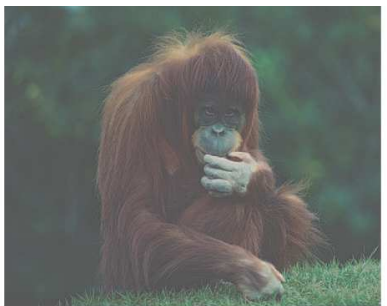

Winner 2

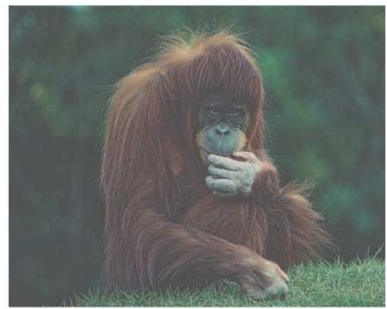

Zolliker

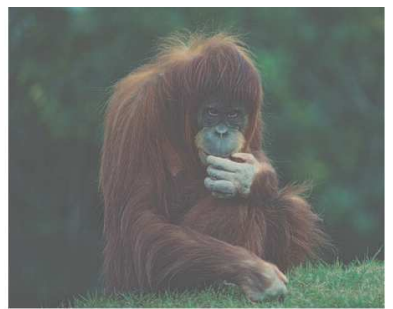

Winner 3

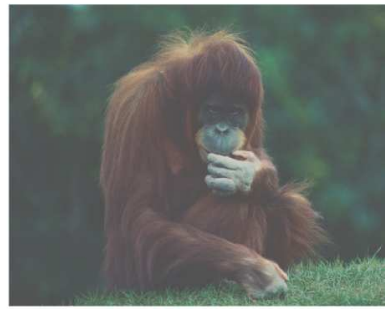

No enhancement

Figure 7. One image transformed with the winners of tier one, as well as Zolliker and no contrast enhancement. 

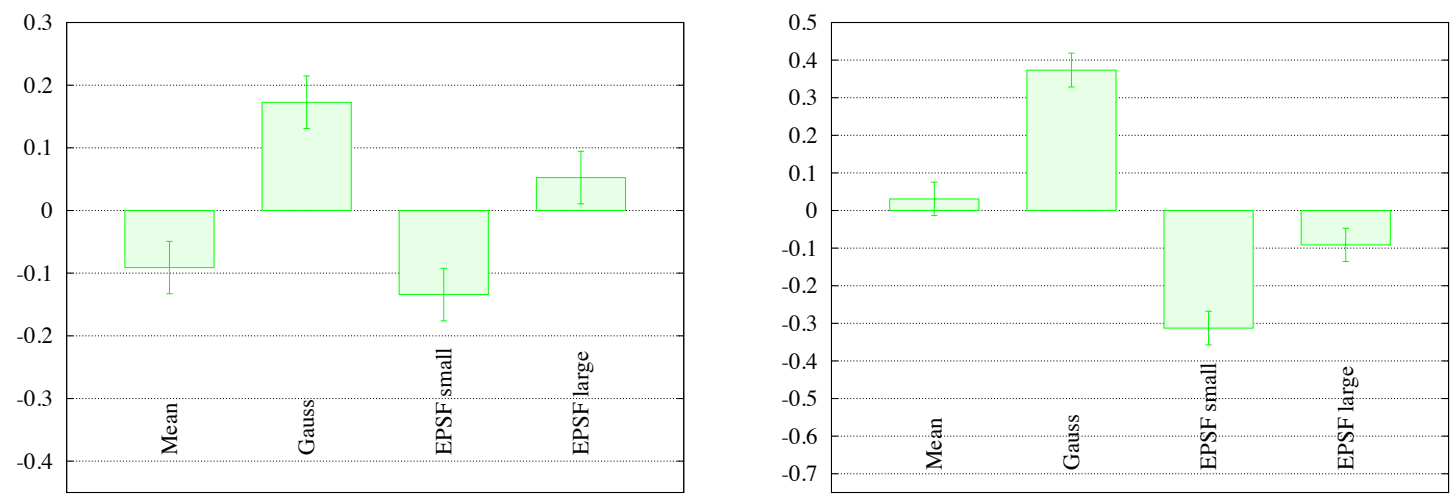

Figure 8. Left: Test 1. Right: Test 2
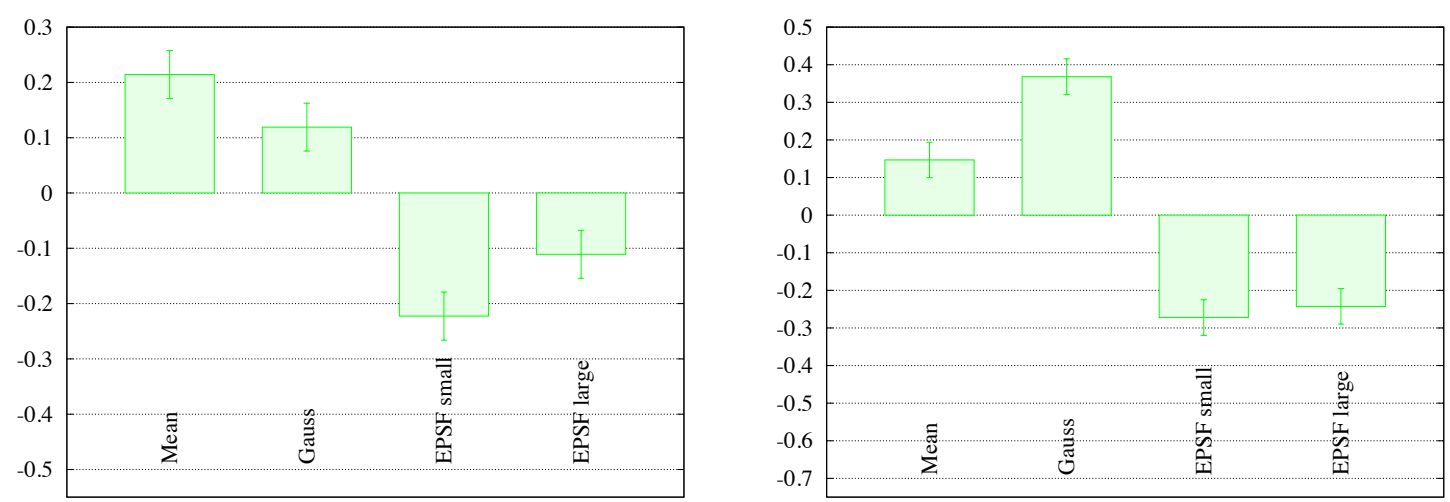

Figure 9. Left: Test 3. Right: Test 4

was the subject of a few previous papers, e.g. ${ }^{12,13}$ Usually, the best performing measure was Structural Similarity Index (SSIM). Hence we considered also SSIM in our discussion about correlation between image quality measures and observer preferences.

In the final phase in our experiment, we were testing an algorithm described in section 4 using detail reconstruction proposed by Zolliker, ${ }^{14}$ the same algorithm without detail reconstruction and the winning parameter-settings of each test from the first phase. Thus, in the final test, not only details enhancement methods were different, but also the underlying algorithms. Hence we were not expecting such a strong correlation between the results of psycho-visual test and $\Delta L C$ measure as in the first phase.

\subsection{Definitions}

Let us remind the formulae for considered image quality measures. We compare two images $X$ and $Y$ with $n \times m$ pixels. Every pixel $x_{i j} \in X$ and $y_{i j} \in Y$, respectively, defines a color coordinate. We are using here the lightness coordinate $L$ in CIELAB color space. If not stated otherwise, we do not distinguish in our notation between a pixel and the color coordinate considered at this pixel.

SSIM. The Structural Similarity Index was introduced by Wang et. al. ${ }^{15}$ and is defined on quadratic image patches of size $k \times k$ at the same location within image $X$ and $Y$. We computed SSIM for the L coordinate in CIELAB color space. Let $P_{X} \subset X$ be such a patch and $P_{Y}$ the corresponding patch for $Y$. We compute the following quantities for the patches:

$$
\bar{P}_{X}=\frac{1}{k^{2}} \sum_{x \in P_{X}} x, \quad \bar{P}_{Y}=\frac{1}{k^{2}} \sum_{y \in P_{Y}} y,
$$




$$
\begin{aligned}
\sigma_{P_{X}}^{2} & =\frac{1}{k^{2}-1} \sum_{x \in P_{X}}\left(x-\bar{P}_{X}\right)^{2}, \\
\sigma_{P_{Y}}{ }^{2} & =\frac{1}{k^{2}-1} \sum_{y \in P_{Y}}\left(y-\bar{P}_{Y}\right)^{2}, \text { and } \\
\sigma_{P_{X} P_{Y}} & =\frac{1}{k^{2}-1} \sum_{i=1}^{k^{2}}\left(x_{i}-\bar{P}_{X}\right)\left(y_{i}-\bar{P}_{Y}\right)
\end{aligned}
$$

The Structural Similarity Index is then defined as

$$
\operatorname{SSIM}\left(P_{X}, P_{Y}\right)=\frac{\left(2 \bar{P}_{X} \bar{P}_{Y}+c_{1}\right)\left(2 \sigma_{P_{X} P_{Y}}+c_{2}\right)}{\left(\bar{P}_{X}^{2}+\bar{P}_{Y}^{2}+c_{1}\right)\left(\sigma_{P_{X}}^{2}+\sigma_{P_{Y}}^{2}+c_{2}\right)},
$$

with two constants $c_{1}$ and $c_{2}$. As proposed by Wang et. al. ${ }^{15}$ we used $c_{1}=1$ and $c_{2}=9$ and $k=8$ for the patch size.

The image quality measure $Q_{\operatorname{SSIM}}(X, Y)$ can be defined as the Structural Similarity Index SSIM averaged over all possible $k \times k$ patches in the images $X$ and $Y$. The resulting measure is in the range $[-1,1]$, and the higher the $Q_{\text {SSIM }}$ value, the more similar are the compared images.

$\Delta L C$. The image quality measure $Q_{\Delta L C}$, described in, ${ }^{16}$ is based on a local contrast measure. We choose the Michelson contrast, see, ${ }^{17}$ as our measure of local contrast. We compute the Michelson contrast on a $k \times k$ patch $P_{X} \subset X$ of the image $X$ as follows (we were using $k=5$ in our experiments):

$$
L C\left(P_{X}\right)=\frac{x_{\max }-x_{\min }}{x_{\max }+x_{\min }}
$$

where $x$ is the luminosity coordinate in CIELAB (at pixel $x \in P_{X}$ ), and $x_{\max }$ and $x_{\min }$ are the highest and the lowest value, respectively, of this intensity on the patch $P_{X}$. Analogously, we can compute the value $L C\left(P_{Y}\right)$ for the corresponding patch $P_{Y}$ in image $Y$, and define

$$
\Delta L C\left(P_{X}, P_{Y}\right)=\left|L C\left(P_{X}\right)-L C\left(P_{Y}\right)\right| .
$$

The image quality measure $Q_{\Delta L C}(X, Y)$ is then finally defined as the measure $\Delta L C$ averaged over all possible $k \times k$ patches in the images $X$ and $Y$.

\subsection{Results}

The correlation between the ordering of the algorithms according to the psychovisual test and the ordering obtained by applying SSIM and $\Delta L C$ is presented in Table 1 . We can see, that the correlation is high in all considered tests. In the first phase (different filters applied to one algorithm), $\Delta L C$ correlates even slightly better than SSIM. Though let us remind, that in this test local contrast was the most important factor tested and we expected high correlation between $\triangle L C$ and observers preferences.

Nevertheless, the ordering by the evaluation of the psycho-visual data methods is not the same as computed using image quality measures. There are a few possible reasons of that. Observers were asked to choose the better image, without showing the reference image. Considered image quality measures compute the difference between two images. We applied it to the original and the mapped image. This means, we assume, that the original is an optimal version of the image and the more similar the mapped image to the original, the better. It does not have to be coherent with observers preferences. Let us also notice, that some mapped images without detail enhancement can look more pleasant or aesthetic, because of their smoothness, even if some details are lost. Additionally, the considered image quality measures do not detect artifacts, which have an influence on observers preferences.

In the second phase the ordering of algorithms obtained from psycho-visual data correlates slightly stronger with the ordering obtained by SSIM, but the correlation with $\Delta L C$ ordering is also relatively high (compare Table 1). This was expected, as in this test not only details enhancing was different in different mapping, but also SSIM takes into account other factors as well. 


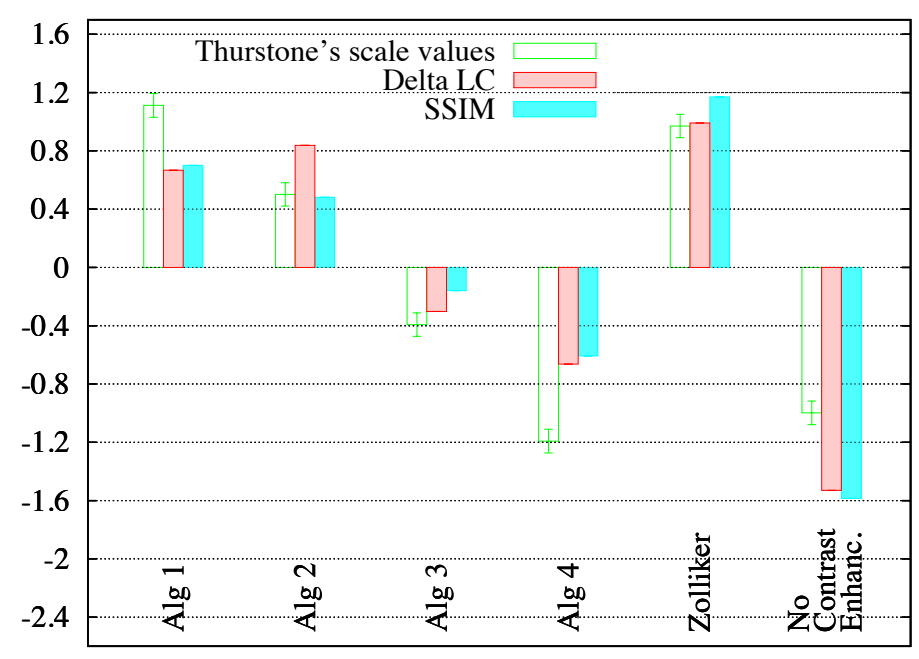

Figure 10. Scale values obtained by Thurstone's method, SSIM values and $\Delta L C$ values for 6 algorithms considered in final test. Scale values, SSIM and $\Delta L C$ values are rescaled to have mean 0 and standard deviation 1 , so that they are comparable. $\Delta L C$ was additionally multiplied by -1 , so that high values correspond to good algorithms.

Comparing with the results of the psycho-visual test, the most significant difference is between 4th and 6th algorithm (see Figure 10). Let us remind that the 6th algorithm had no details enhancement at all. This had major influence on SSIM or $\Delta L C$ of this mapping, as we lose a lot of details compared with original. Loss of details is not favored also by observers, by similarly to the 4th algorithm, where loss of saturation was higher than in other algorithms. As we mentioned before, we applied SSIM or $\triangle L C$ only to the $L$ coordinate of CIELAB, hence image quality measures could not detect this factor.

\begin{tabular}{|l||c|c|}
\hline Test & $\Delta L C$ & SSIM \\
\hline \hline 1 & 0.67 & 1 \\
\hline 2 & 1 & 0.33 \\
\hline 3 & 1 & 0.67 \\
\hline 4 & 0.67 & 0.67 \\
\hline \hline Final & 0.6 & 0.73 \\
\hline
\end{tabular}

Table 1. Kendall's rank correlation between the ordering of the algorithms obtained in the psycho-visual test and the ordering based on SSIM and $\Delta L C$.

\section{CONCLUSIONS}

We tested several simple filter concepts for contrast preservation in context of gamut mapping. Here, the filtering was applied as pre-step of the actual gamut mapping. Based on a psycho-visual test, we could show that the well-known Gauss filter performed very well in comparison to a subsequent reconstruction of details. Further it was possible to show that this performance analysis agrees with established image quality measures. Because of its simplicity it could be expected that our concept is comparable with an arbitrary gamut mapping. On the other hand some questions remain open, particularly in the context of the multi-scaled gamut mapping approach.

\section{REFERENCES}

[1] Central Bureau of the CIE, Vienna, CIE Publication 156: Guidelines for the Evaluation of Gamut Mapping Algorithms (2004). 
[2] Reinhard, E., Khan, E. A., Akyüz, A. O., and Johnson, G. M., [Color Imaging: Fundamentals and Applications], A. K. Peters, Ltd., Natick, MA, USA (2008).

[3] Li, Y., Sharan, L., and Adelson, E. H., "Compressing and companding high dynamic range images with subband architectures,” ACM Trans. Graph. 24(3), 836-844 (2005).

[4] Mallat, S., [A wavelet tour of signal processing], Academic Press (1998).

[5] Morovic, J. and Wang, Y., "A multi-resolution, full-colour spatial gamut mapping algorithm," in [Color Imaging Conference], 282-287, IS\&T - The Society for Imaging Science and Technology (2003).

[6] Farup, I., Gatta, C., and Rizzi, A., "A multiscale framework for spatial gamut mapping," IEEE Transactions on Image Processing 16(10), 2423-2435 (2007).

[7] Bala, R., deQueiroz, R., Eschbach, R., and Wu, W., "Gamut mapping to preserve spatial luminance variations," Journal of Imaging Science and Technology 45(5), 436-443 (2001).

[8] Eschbach, R., Bala, R., and deQueiroz, R., "Simple spatial processing for color mappings," Journal of Electronic Imaging 13(1), 120-125 (2004).

[9] Zolliker, P. and Simon, K., "Adding local contrast to global gamut mapping algorithms," Third European Conference on Colour in Graphics, Imaging, and Vision(CGIV) 1, 257-261 (2006).

[10] CIE, “Guidelines for the evaluation of gamut mapping algorithms," Tech. Rep. TC 8-03, CIE (2003).

[11] Caluori, U. and Simon, K., "An rgb color management concept based on an improved gamut mapping algorithm," in [Proc. IS\&T/SPIE Symposium on Electronic Imaging], 7241, SPIE (2009).

[12] Hardeberg, J. Y., Bando, E., and Pedersen, M., "Evaluating colour image difference metrics for gamut mapping images," Coloration Technology 124, 243-253 (July 2008).

[13] Bonnier, N., Schmitt, F., Brettel, H., and Berche, S., "Evaluation of spatial gamut mapping algorithms," in [14th Color Imaging Conference], 14, 56-61, IS\&T/SID (2006).

[14] Zolliker, P. and Simon, K., "Retaining local image information in gamut mapping algorithms," IEEE Transactions on Image Processing 16, 664-672 (March 2007).

[15] Wang, Z., Bovik, A., Sheikh, H., and Simoncelli, E., "Image quality assessment: From error visibility to structural similarity," IEEE Transactions on Image Processing 13, 600-612 (April 2004).

[16] Baranczuk, Z., Zolliker, P., and Giesen, J., "Image quality measures for evaluating gamut mapping," accepted to 17th Color Imaging Conference (2009).

[17] Michelson, A. A., [Studies in Optics], U. Chicago Press (1927). 